\title{
A Study of Users' Experiences and Beliefs about Software Update Messages
}

\author{
Michael Fagan ${ }^{a *}$ Mohammad Maifi Hasan Khan ${ }^{a}$ \\ and Ross Buck ${ }^{b}$ \\ ${ }^{a}$ Department of Computer Science and Engineering; ${ }^{b}$ Department of \\ Communications
}

University of Connecticut 



\begin{abstract}
Prior studies report that computer users are often confused by software update messages. This study complements prior efforts by examining the relationship between self-reported ratings of specific software types and users selfreported measure of confusion, annoyance, and message noticeability for different software types using data gathered via two surveys $(\mathrm{N} 1=78, \mathrm{~N} 2=172)$ from anonymous computer users. The survey results showed that participants are commonly hesitant to apply updates, even though they report caring a lot about computer security and privacy, a trend which applies to many different software. Annoyance and confusion with messages were common complaints from our participants. Also, the level of reported annoyance and/or confusion with a software's messages proved a predictor of participants' hesitation in applying the updates for some software we tested. Finally, we use data gathered as participants viewed real-world update messages to highlight and explain some "good" and "bad" update/warning messages as well as draw connections between the emotions annoyance, confusion, importance, and noticeability.

Keywords: update message design; affective-cognitive design; user opinions; user experiences
\end{abstract}

\title{
1. Introduction
}

As the usage of computers and the Internet continues to grow, launching security attacks remotely using various techniques such as baiting, click-jacking, cross-site scripting, phishing scams, and spoofing is becoming much easier and more profitable $[26,18,1]$. Interestingly, cyber criminals often exploit known software vulnerabilities to breach a computer's security $[26,1,12]$. These kinds of attacks are often preventable by keeping systems up to date by applying the latest software and security updates [4, 28].

Unfortunately, while software companies are constantly trying to identify security vulnerabilities and release fixes promptly, prior studies showed that users are often confused by software update messages, and ignore recommended updates [30, 29]. While a limited number of prior studies identify lack of understanding and the prevalence of poor wording/technical jargon [13, 30, 29] as some of the reasons behind users' behavior, we would like to argue that human factors such as personal attitudes, beliefs, and specific software type may change the effectiveness of software update messages as well. Specifically, prior studies mostly focus on comprehension of software update messages and the underlying update mechanism and do not focus on the emotional aspect of the communication process, which may affect users' software update behavior significantly.

To investigate these phenomena, we designed and executed a study to investigate user attitudes and perceptions regarding software update and warning messages. Specifically, we ask the following research questions:

1. What are the common behaviors and attitudes of users related to software update and warning messages? 
2. How does concern regarding computer security, performance, and privacy effect users software update/warning behaviors and attitudes?

3. Does users update/warning behavior and attitudes, including frequency vary between software types?

4. How prevelant is annoyance, confusion, importance, and noticeability in response to update/warning messages and what implications could this have on update frequency?

5. What is the relationship between emotions felt due to update/warning messages and application of updates?

To answer the above questions, our study utilizes two frameworks, namely, the Communication-Human Interface Processing (C-HIP) framework [6] and the AffectReason-Involvement (ARI) framework [3], as tools to identify the strengths and weaknesses of the messages from an affective-cognitive perspective. These models lead the development of two surveys distributed to anonymous computer users in Sept. of $2013(\mathrm{~N}=78)$ and Feb. of $2014(\mathrm{~N}=172)$ respectively.

Our analysis considers several variables: software type, concern regarding computer security, performance, and privacy, user's software update/warning behaviors and attitudes, update notification frequency, update application delay, and emotions elicited by update/warning messages. All of these variables are defined in our study using survey instruments and self reported ratings that reflect each variable. Details of variable definitions can be found in Section 3.3.

Using the variables mentioned above, our analysis finds that participants are commonly hesitant to apply updates, despite high reports of care about computer security and privacy. This trend applies to many different software. Annoyance and confusion with messages were found to be common complaints among our participants, and for some software in our sample, the level of reported annoyance and/or confusion with a software's messages is a predictor of participants' hesitation in applying the updates. We also present image-based findings extracted from participants' responses gathered while they viewed realworld update/warning messages. These findings show more connection between annoyance and confusion, as well as connection between importance and noticeability. Finally, we use the image-based data to highlight and explain some "good" and "bad" update/warning messages.

The rest of the paper is organized as follows. The Related Work section presents the basic concepts underlying our analysis and describes prior research that is related to ours. The Methods section explains the design of our data collection policy and mechanism used in this paper. In the Evaluation section, we present our findings and relate them back to our paper's central argument. In the Discussion section, we discuss the limitations of our study along with future directions. Finally, the Conclusion section concludes the paper. 


\section{Related Work}

While a large volume of research exists on risk communication and designing warning messages for various products in other domains such as poisons, cigarettes, fire extinguishers, cars, and industrial equipment $[2,27,31,33,36$, 38,7 , only a handful of prior work have investigated the challenge of designing effective software warning and update messages.

Among these, one recent work investigated the readability of computer warning messages, and reported that, on average, a user needs to have at least 10 years of education to understand the presented messages [13]. This indicates a high probability of confusion among many users when reading the messages (i.e., those without adequate education). Some work suggests that users becomes "bored" with complex messages and give them less attention. A study of users and software warning and security messages showed attention, as well as effects on attitudes and beliefs are tied to the linguistic complexity of the messages [14].

With this connection in mind, we also draw attention to two other works that identified issues with user comprehension in the domain of computer software. First, a team found low attention and comprehension rates among users (e.g., only $17 \%$ paid attention to permissions, and only $3 \%$ showed full comprehension of the messages) of Android permissions when installing new apps [8]. Additionally, a more recent investigation found large differences between actual changes made by updates and the changes the participants thought were being made [30]. We argue that this failure to grab attention and effectively communicate the intended information falls on the software messages and that identifying why users do not pay attention to and understand the messages is a key goal towards improving them since without this information, a user cannot decide to apply or not apply an update based on pertinent data and instead must rely on other information and instincts.

All of these issues have inspired investigations into how to fix or avoid this disconnect between users and software companies. Several efforts have looked into effective notification strategies [22, 24, 29], but these studies mostly focus on how to manage the notification system and do not focus on the design of the notification message itself. An applicable lesson from these projects is that past experiences are very important to decision making when it comes to software updates [29]. Specifically, they find that bad experiences with updates will reduce the likelihood of a user applying updates from that software in the future.

Though delivering messages in the best possible way is certainly important to adherence, the design and communication potential of those messages is also important. One notable attempt at introducing more radical affective-cognitive appeals showed users real-life mobile data security risks (i.e., what information from the phone will be available based on the apps' permissions) when they installed apps to make users more aware of privacy concerns [15]. This study found that the information made users pay more attention to these issues, showing how the design of messages can be used to better communicate information important to software security and functionality to users. 
Our work builds on this wealth of prior research and investigates the effect of software update message and type on the level of annoyance, confusion, and users' software update behavior by conducting an extensive, multi-phased study.

\section{Methods}

As we want to investigating the role of users attitudes, beliefs, and emotions in the effectiveness of software update messages and users update behavior, two behavioral models that are widely used in marketing research to design and evaluate advertisements and warning messages [34, 32] were chosen to help design the survey and understand the results. The models used are the Communication-Human Information Processing (C-HIP) model [6], and the Affect-Reason-Involvement (ARI) model [3]. Brief overviews of both models along with the design of the survey are given below.

\subsection{Communication-Human Information Processing (C-HIP) Model}

The Communication-Human Information Processing (C-HIP) protocol [6] is widely used by the marketing research community to determine, where, if at all, the failure in persuasion happens when trying to warn a user and convince them to perform a behavior. A visual representation of the C-HIP framework can be found in Figure 1(a). Specifically, C-HIP models the multiple information processing steps that are often involved in the decision making process. In the C-HIP model, the source is the entity that attempts to communicate the message through one or more communication channels (e.g., visual, auditory) to one or more receivers (i.e., human). In our work, the source is the software company, the channel is the software warning/update message, the receiver is the computer user, and the expected behavior is the user applying the update.

According to the C-HIP model, for a update/warning message to be effective, first, the message must be sufficiently attention grabbing. In other words, the message must acquire attention upon first encounter and it must retain that attention long enough for the user to comprehend the message. Next, once the basis of attention has been created and maintained, the receiver must understand the information being transmitted. Thus, the model assumes that any message that cannot be understood will be less likely to cause the receiver to execute the behavior. Once the receiver understands the message, the message must also align with the receiver's beliefs. If the information from the source and the receivers attitudes or beliefs about the topic at hand do not match up and the information fails to convince the user to breach his/her beliefs and attitudes, the model predicts a failure at the attitudes and beliefs stage. Finally, if the receiver sees, reads, understands, and agrees with the message, the final job of the message is to motivate the user into action. This could mean highlighting the desired choice or convincing the user that the desired action is important. 
Given the multi-stage breakdown of the information processing steps involved in the decision making, in our work, we use the C-HIP model as an investigative tool to design the survey questions that may help us to identify the stage(s) where the current software warning/update messages fail.

\subsubsection{Affect-Reason-Involvement Model}

In addition to the C-HIP model, we also use a model of persuasion that considers 3 vectors to behavior compliance. Under the Affect - Reason Involvement (ARI) model [3], a user can be convinced via an emotional appeal, a rational appeal, or both. The compliance represents the behavioral success of the message. In our case, messages may cater to both, one, or none of the appeals and still see either possible behavioral outcomes (i.e., compliance vs. ignoring). Figure 1(b) shows the visual representation of this model.

In the ARI model, rational appeals attempt to convince a user via logical means. An example of a rational appeal would be a message that describes exactly which problems the update is going to fix and explain why those fixes are needed. In contrast, emotional appeals function by stirring emotions in users related to the compliance with the desired behavior. An example of an emotional appeal would be a message that displays a large, ominous warning image while warning users regarding the possibility of loss of data due to failure to update the system. Emotional appeals work by tapping into a user's emotional subconscious.

In our study, we collect data that attempts to measure users attitudes, beliefs, and motivation along with emotional and rational appeals created by update messages and correlate the prevalence of each appeal with possible behavioral responses indicated by the user.

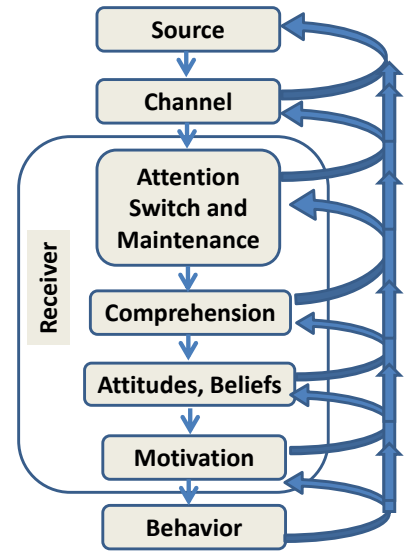

(a)

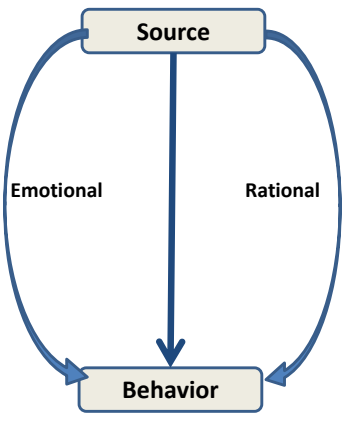

(b)

Figure 1: (a) The Communication-Human Information Processing (C-HIP) Model [6] , (b) The Affect-Reason-Involvement (ARI) Model 


\subsection{Survey Design}

In Sept. of 2013, a short, strictly quantitative, uncompensated survey was distributed. In Feb. of 2014, an extended version of the short survey was administered where each participant was given a $\$ 15$ (USD) Amazon gift card for participation. This survey included the questions from the short survey, as well as quantitative questions regarding specific software packages and updates. It also included another section where participants were asked to give feedback on images of existing update/warning messages. More details on the design and administration of these two surveys are given below.

\subsubsection{Short Survey Questions}

Survey takers were first asked some background questions for demographic and analysis purposes. These questions asked participants their gender, age, and experience with various software packages. A listing of background questions from the short survey can be found in Table 2 .

The short version of the survey also asked respondents about their experiences with update messages in general. Since keeping software up to date is important to computer performance, security, and by extension the privacy of the user using the computer, we attempted to gauge respondent's opinions about these issues in relation to software updates in general.

The C-HIP and ARI models were considered while designing survey questions. For example, both models suggest that if someone feels something is annoying, this will make them pay less attention to the message and less likely to adhere. Thus, we asked users if they had previously been annoyed by messages to tap into this aspect of the decision making process. The other questions were designed similarly. Questions regarding participants' opinions and experiences that were included in this survey, along with the corresponding C-HIP and ARI designations are shown in Table 1 . The designations in Table 1 indicate which stage/aspect of each model the question is targeted at. Most questions try to hone in on specific aspects of each persuasion model. All columns are covered except the Attention stage of the C-HIP model due to the fact that attention appears to be quite difficult to measure accurately via an online survey due to the possibility of bias introduced by the fact that they user is not viewing the image "naturally" (i.e., in a pop-up while using the computer). Formal definitions of all the variables considered in analysis are presented in Subsection 3.3.

\subsubsection{Extended Quantitative Questions}

To better analyze software specific attitudes and experiences, the additional quantitative questions added to the short survey for the second round focused on specific software products. Participants were first asked to identify from a list which software packages they use or have recently used. Table 3 shows the list of software respondents were shown to choose from.

The list of software provided by the respondent was used to generate the remainder of the extended survey questions. For each software package selected by the participant, they were directed to a survey with questions asking about their 
1 How much do you worry about your computer's 1 security?

${ }_{2}$ How much do you care about keeping your software 2 up to date?

3 How much do you worry about your computer's privacy?

On average, how long would you say you wait before

4 applying a software update after seeing the notification for the first time?

5 Are you worried that not keeping your software up

5 to date will hurt your computer's speed?

6 Are you worried that not keeping your software up

to date will hurt your privacy?

7 Are you worried that not keeping your software up

7 to date will hurt your computer's security?

8 Do you generally know what changes updates make to you computer and software?

9 Have you ever applied a software update that you

9 later found out was malicious?

${ }_{10}$ Have you ever not applied an update because you

thought it was fake or malicious?

11 Have you ever been annoyed by an update message?

12 Have you ever been confused by an update message?

13 Have you ever told a friend or colleague that they

13 should apply an update?

14 Have you ever been hesitant to apply an update?

15 What do you think is the PRIMARY motivation for

15 software companies to release software updates?

$\mathrm{x} \quad \mathrm{x}$

$\mathrm{X}$

$\mathrm{x} \quad \mathrm{x}$

$\mathrm{x} \quad \mathrm{x}$

$\mathrm{x}$

$\mathrm{x}$

$\begin{array}{lllll}\mathrm{X} & \mathrm{X} & \mathrm{X} & \mathrm{X} & \mathrm{x}\end{array}$

$\begin{array}{lllll}\mathrm{x} & \mathrm{X} & \mathrm{X} & \mathrm{x} & \mathrm{x}\end{array}$

$\mathrm{x} \quad \mathrm{x}$

$\mathrm{X}$

\begin{tabular}{l|l}
$\mathrm{x}$ \\
$\mathrm{x}$
\end{tabular}

$\mathrm{x}$

$\mathrm{x}$

$\mathrm{x}$

Table 1: Short survey questions and vorresponding C-HIP categories and ARI vectors.

opinions and experiences with that software and its update messages. These survey questions followed a template to allow comparison between packages. The template is shown in Table 4. This table also includes, where applicable, a mapping of the question to the appropriate element(s) of the C-HIP and ARI models.

In each survey, the word "BLANK" was replaced with a software package name and the question's grammar was altered, as necessary, for correctness. These questions were developed using the same strategy as for the short survey and were influenced by the responses from the first stage of the study. 


\begin{tabular}{cll}
\hline$\#$ & Question & Notes \\
\hline 1 & What is your gender? & \\
2 & What is your age? & \\
3 & $\begin{array}{l}\text { For each of the following, rate on a scale of } \\
1 \text { to } 7 \text { how knowledgeable you are in using }\end{array}$ & $\begin{array}{l}\text { Licrosoft Windows, Apple } \\
\text { iPhone, Android }\end{array}$ \\
& $\begin{array}{l}\text { of each software or device. } \\
4\end{array}$ & $\begin{array}{l}\text { How much do you worry about your com- } \\
\text { puter's security? }\end{array}$ \\
5 & $\begin{array}{l}\text { How much do you care about keeping your } \\
\text { software up to date? }\end{array}$ \\
6 & $\begin{array}{l}\text { How much do you worry about your com- } \\
\text { puter's privacy? }\end{array}$
\end{tabular}

Table 2: Background questions asked of participants. Note: $1=$ Not at all, $7=$ Very much.

\subsubsection{Image Based Survey}

Finally, the extended version of the survey also included a section that displayed specific images of mostly update messages. It should be noted that one of the images shown depicted a virus notification message from an anti-virus software. Though this is not an update message, it shares many characteristics with software update messages such as similar size and layout, affiliated concerns (e.g., computer security, computer performance), and similar desired behavior (e.g., for users to click a button to remove the virus).

Respondents rated their perception of four aspects of each update/warning, based on each image. In all, respondents were shown 14 images. All 14 images can be seen in the Appendix. The questions that accompanied each image can be seen in Table 5 . Please note that the two qualitative questions in Table 5 (i.e., question \# 5 and \# 6) are not analyzed for this report.

\subsection{Definition of Variables}

Our analysis considers several variables: user background, software type, concern regarding computer security, performance, and privacy, software update/warning behaviors and attitudes, update notification frequency, update application delay, and emotions elicited by update/warning messages. All of these variables are operationally defined in our study using survey instruments and self-reported ratings that reflect each variable.

Participants' background is ascertained by the demographic questions (i.e., questions 1-3) found in Table 2 and all questions defined in Table 1. For participants who took our extended survey, their background was also measured by the software they reported commonly using and the responses to questions 1-3 of each software specific survey, as described in Table 4. Concern regarding computer security, performance, and privacy are all measured using self-reported ratings, specifically questions 4-6 in Table 2. Update notification frequency and application delay are also measured using self-reported scales, namely questions 4 and 5 , respectively from Table 4 for each software. 


\begin{tabular}{lc}
\hline Software & Category \\
\hline Microsoft Windows & Operating System \\
Apple laptops or desktops & Operating System \\
Linux & Operating System \\
iPhone & Mobile OS \\
Android & Mobile OS \\
Mozilla Firefox & Web Browser \\
Google Chrome & Web Browser \\
Internet Explorer & Web Browser \\
Safari & Web Browser \\
Microsoft Office & Productivity Software \\
OpenOffice & Productivity Software \\
Adobe Acrobat & Productivity Software \\
Libre Office & Productivity Software \\
iTunes & Media Software \\
QuickTime & Media Software \\
Windows Media Player & Media Software \\
VLC & Media Software \\
Skype & Communication Software \\
Norton products & Security Software \\
McAfee products & Security Software \\
Malwarebytes & Security Software
\end{tabular}

Table 3: Software packages included in extended survey.

Three emotions, annoyance, confusion, and boredom as they relate to each software's messages are defined using the ratings for statements 6,7 , and 8, respectively from Table 4 . Emotions evoked by specific messages (i.e., annoyance, confusion, importance, and noticeability) are defined using questions 1-4 in Table 5. In this case, the emotion mentioned in the question is the emotion that question is used to measure.

Software type for each software as defined in our study is given in Table 3.

\subsection{Procedure}

The short survey was hosted online and was accessible to the public via the Internet. Since the first round of the study featured a short survey, all questions were shown to the participant on two pages. The first page asked the respondent to answer the background questions shown in Table 2. Once completed, the participant was then directed to the second page which featured the remaining questions as presented in Table 1.

In the extended survey, due to the increased length of the survey, participants were required to create an account before beginning. This allowed participants to leave in the middle of the survey and log back in later at their convenience. Respondents also provided email addresses for the purpose of compensation. Basic information, including all the short survey questions was shown to the 
1 How long have you used BLANK?

2 How many hours a day do you use BLANK?

3 On a scale of 1 to 7 , rate your opinion of BLANK. 1

$=$ very negative opinion, $7=$ very positive opinion Approximately how often do you see an update 4 message for BLANK? Please note, we are asking 4 about updates for BLANK, not other software. If you are confused, please select I Don't Know

5 Approximately how long after you see a BLANK 5 update message do you wait to apply the update? On a scale of 1 to 7 , rate how much you agree with each statement. $1=$ do not agree at all, $7=$ agree completely.

6 BLANK update messages are annoying.

7 BLANK update messages are confusing.

8 BLANK update messages are boring.

$x$

Table 4: Extended survey questions and corresponding C-HIP categories and ARI vectors.

\begin{aligned} \hline$\# &$ Question \\ \hline 1 & How important is the message? (rate from $1=$ not at all, to $7=$ very) \\ 2 & How annoying is the message? (rate from $1=$ not at all, to $7=$ very) \\ 3 & How confusing is the message? (rate from $1=$ not at all, to $7=$ very) \\ 4 & How noticeable is the message? (rate from $1=$ not at all, to $7=$ very) \\ 5 & What did you like about the message? \\ 6 & What did you dislike about the message? \end{aligned}

Table 5: Questions shown with update images.

user when creating an account. Once an account was created, the system asked individuals to select which software packages he/she uses or has recently used. Software specific surveys were then generated as described in section 3.2.2. Once these surveys were completed, respondents were shown the image based questions as described in section 3.2.3. Both studies were approved by the University Institutional Review Board.

Participation in both survey rounds was voluntary. Respondents were allowed to stop participation at any time during the study.

\subsection{Sampling Methodology}

Participants who responded to the short survey were recruited using the snowball sampling method [11]. As an initial seed, a group of 5 colleagues 
and friends were selected and directly contacted by the researchers. These individuals varied in occupation, age, sex, and geographic location. The initial seed was encouraged to share the survey with others. Using this sampling method, we were able to get 78 participants. The data from 71 of those responses are used in our analysis due to the data from 7 participants being incomplete. Due to the brevity of this survey, no compensation was offered. The survey was run during a 1 week period in Sept. of 2013.

For the second phase of the study, a flyer was distributed through university and community email lists. Interested participants were directed to a survey website hosted by the authors. The website was open for two days in Feb. 2014 and in that time, we gathered 172 sets of responses. Of those, 155 were complete and were used in our analysis.

\section{Evaluation}

This section details the findings from the online questionnaire that was distributed twice, in two separate rounds, as explained in section 3. In our discussion, findings are referred based on the period during which the data was gathered. Specifically, the first phase was conducted in Sept. 2013, and the second phase was ran in Feb. 2014. The data from all these participants was used, though some missing answers are noted as necessary in the findings. Where applicable, results are discussed with regards to the prior work described in section 2 that is closely related to our study.

\subsection{Sample Demographics}

The Sept. 2013 sample tends towards 20 to 30 year olds with a median of 26 years old. The average respondent age is 33 with a standard deviation of 14 years. The Feb. 2014 sample population is younger than the first phase's with a median of 21 years old. The average age is 22 with a standard deviation of 5.4 .

The first sample has a slight male bias. Overall, $58 \%$ of the first sample is male, and $42 \%$ is female. In our second sample, the bias is flipped. $60 \%$ of the respondents is female and $40 \%$ are male.

Please note that, despite the difference in sample size, demographics, and compensation scheme, the findings from both surveys for the common questions showed similar distributions (see Figure 10). More regarding the limitations of our study can be found in Section 5 .

\subsection{Lack of Understanding}

In both surveys, participants were asked to rate their concern about three computing issues related to update messages, specifically, computer security, keeping software up to date, and computer privacy. Table 6 shows the average of each rating from both samples. In general, most respondents care about all 3 issues, but privacy seems to be a bigger concern than keeping software up to date and security in general. This is interesting since software updates are 
Sept. $2013(\mathrm{n}=71) \quad$ Feb. $2014(\mathrm{n}=155)$

\begin{tabular}{rllll}
\hline Question Avg. & \multicolumn{2}{c}{ St. Dev. } & Avg. & \multicolumn{2}{c}{ St. Dev. } \\
\hline Security & 5.0 & 1.7 & 4.8 & 1.7 \\
Keep up to date & 5.1 & 1.4 & 4.9 & 1.6 \\
Privacy & 5.6 & 1.4 & 5.2 & 1.5
\end{tabular}

Table 6: Response statistics from both phases for questions asking participants to rate (from 1 to 7 , with $1=$ none at all and $7=$ extremely concerned) their concern about 3 issues.

critical to both privacy and security. It shows a lack of general understanding regarding the critical relation between software updates and computer security and privacy.

These ratings, in the context of the C-HIP model, tap into a critical deficiency in attitudes and beliefs of our participants, which may lead to reduced levels of motivation. Thus, to be successful, an update message needs to contain information explaining to users that security and privacy are both tied to software updating and that applying the update will help both privacy and security (when applicable).

\subsection{Overall Prevalence of Hesitation}

Both samples have a history of feeling hesitant about applying an update. Figure 2 shows a summary of the responses to the question "Have you ever been hesitant to apply an update?" for both samples of the study.

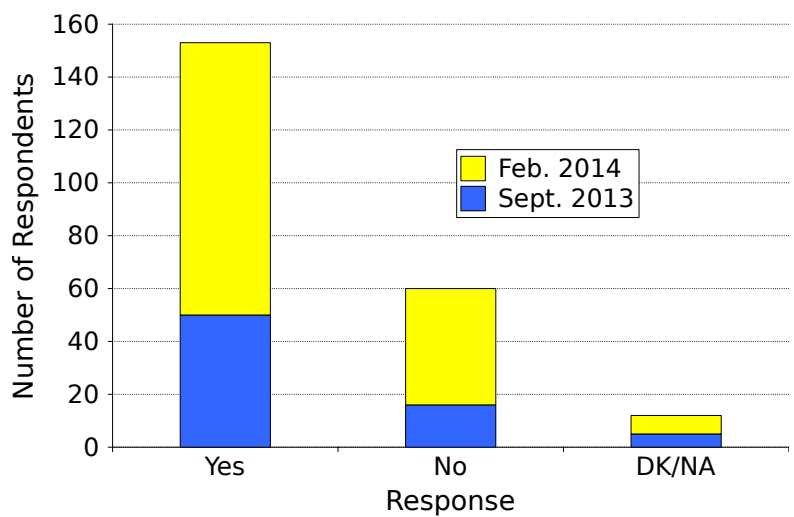

Figure 2: Responses from both phases for "Have you ever been hesitant to apply an update?" Note: $\mathrm{DK}=$ Don't Know, NA = No Answer

If a respondent indicated that they felt hesitant, they were asked to identify why. In the first phase of the study, of the 50 who responded yes, 45 left an explanation. In the second run of the survey, 88 of the 103 "Yes" respondents left a comment. A majority of participants, in both rounds report feeling hesitant because they didn't know what the update was going to do, worried the update was fake or malicious, and/or worried that the update would hurt their system's 
performance. In the first phase, these reasons are identified in $69 \%$ of the comments left. In the second phase, $62 \%$ of the comments indicates at least one of these reasons.

The reasons indicated by participants to explain their hesitation to apply updates suggest a failure at Attitudes-Beliefs because they delay updates because they believe it will hurt their system. Our participants' propensity for skepticism regarding software updates follows the findings of [30]. In that study, the authors demonstrated that participants commonly had misunderstanding about what an update is changing in their system, leading to frustration. Our results are a projection of this phenomenon since the frustration related to misunderstandings can result, at least in part to the large portion of our samples not trusting updates.

Another pattern found in both sets of comments is the issue of devoting time and effort to the update. In both rounds, approximately $16 \%$ of respondents who left comments said they didn't want to devote time to applying the update or were busy and unable to devote the time. In other words, if users are unwilling to devote time to applying an update, this indicates that in some cases updates are viewed as unimportant.

In the context of both the C-HIP and ARI models, our participant's hesitancy can be viewed as a failure of the update messages to convince them to perform the action of updating. By the ARI model, it is likely that update messages do not adequately incorporate appropriate rational and emotional appeals.

This finding relates to one of the findings of another work that showed users sometimes had to assess if an update was "worth" the time to apply [29]. Our survey results indicate that, at least some of the time, participants didn't apply updates because they deemed the update not "worth it."

\subsection{Hesitation Extends to Many Software}

The prevalence of hesitation is also apparent in the data collected about specific software packages and messages. Table 7 shows the distribution of the aggregation of all software based surveys for the question "Approximately how long after you see a $B L A N K$ update message do you wait to apply the update?," where $B L A N K$ is replaced with each software name as well as the response distributions for most of the softwares asked about.

Of all software-based responses, participants report not applying updates at all $28 \%$ of the time. Behavior varies between software packages, though. At $62 \%$, Apple's Safari has the highest percentage of respondents who reported that they do not apply updates for that software. The breakdown of responses for Safari can be seen in Table 7 .

On the other side of the spectrum is the iPhone. $72 \%$ of participants who use the iPhone report applying updates within a week or less (apply the update within a week, 3 days, 1 day, or immediately). The full breakdown of the responses for the iPhone can be found in Table 7 .

Based on these observations, and knowing that both the iPhone and Safari are made by Apple Inc., the difference in compliance rate suggest that software 


\begin{tabular}{rcccccc}
\hline & & \multicolumn{5}{c}{ Within... } \\
Software & Immediately & 1 day & 3 days & a week & a month & Never \\
\hline Windows & $11 \%$ & $24 \%$ & $13 \%$ & $23 \%$ & $16 \%$ & $13 \%$ \\
Apple PC & $5 \%$ & $16 \%$ & $7 \%$ & $30 \%$ & $39 \%$ & $4 \%$ \\
iPhone & $21 \%$ & $21 \%$ & $22 \%$ & $11 \%$ & $18 \%$ & $6 \%$ \\
Android & $21 \%$ & $18 \%$ & $18 \%$ & $12 \%$ & $18 \%$ & $15 \%$ \\
Firefox & $26 \%$ & $17 \%$ & $7 \%$ & $9 \%$ & $17 \%$ & $24 \%$ \\
Chrome & $14 \%$ & $20 \%$ & $11 \%$ & $8 \%$ & $4 \%$ & $43 \%$ \\
Internet Explorer & $9 \%$ & $0 \%$ & $19 \%$ & $9 \%$ & $25 \%$ & $38 \%$ \\
Safari & $4 \%$ & $8 \%$ & $6 \%$ & $12 \%$ & $8 \%$ & $62 \%$ \\
MS office & $17 \%$ & $16 \%$ & $10 \%$ & $12 \%$ & $15 \%$ & $30 \%$ \\
Acrobat & $13 \%$ & $15 \%$ & $10 \%$ & $13 \%$ & $18 \%$ & $31 \%$ \\
iTunes & $13 \%$ & $10 \%$ & $7 \%$ & $13 \%$ & $32 \%$ & $24 \%$ \\
WMP & $14 \%$ & $16 \%$ & $10 \%$ & $8 \%$ & $6 \%$ & $47 \%$ \\
QuickTime & $12 \%$ & $0 \%$ & $8 \%$ & $16 \%$ & $16 \%$ & $48 \%$ \\
VLC & $14 \%$ & $6 \%$ & $9 \%$ & $9 \%$ & $14 \%$ & $49 \%$ \\
Skype & $20 \%$ & $10 \%$ & $7 \%$ & $14 \%$ & $14 \%$ & $34 \%$ \\
Video Games & $38 \%$ & $12 \%$ & $12 \%$ & $4 \%$ & $10 \%$ & $24 \%$ \\
Norton & $19 \%$ & $19 \%$ & $13 \%$ & $23 \%$ & $3 \%$ & $23 \%$ \\
MacAfee & $11 \%$ & $14 \%$ & $17 \%$ & $14 \%$ & $11 \%$ & $31 \%$ \\
Malwarebytes & $50 \%$ & $19 \%$ & $6 \%$ & $6 \%$ & $6 \%$ & $13 \%$ \\
Overall & $\mathbf{1 7 \%}$ & $\mathbf{1 5 \%}$ & $\mathbf{1 1 \%}$ & $\mathbf{1 3 \%}$ & $\mathbf{1 6 \%}$ & $\mathbf{2 8 \%}$
\end{tabular}

Table 7: Response statistics for "Approximately how long after you see a $B L A N K$ update message do you wait to apply the update?" Softwares with sample size $<15$ respondents (i.e., Linux, OpenOffice, and Libre Office) were omitted due to low sample number.

vendor is not always a predictor of how quickly users will update the vendors' products. While this can be explained by many factors, we noted that Safari is a laptop/desktop application whereas the iPhone is a mobile platform. Safari is also a web browser, while the iPhone is a device/operating system. Since how a user uses both products is distinct, user attitudes and beliefs (as per the C-HIP model) will be different for each device.

After analyzing the extended survey data for each software package, another interesting pattern emerged for both desktop/laptop OSes (e.g., Microsoft Windows) and mobile platforms (e.g., iPhone and Android). In our sample data, the different OSes and different mobile platforms that we asked about seem to have similar correlations between responses to the question about how long the respondent waits to apply a software's update and rating of the respondent's opinions of specific aspects of that software's update messages.

Table 8 shows the correlations (Pearson's r value) between each respondent's rating of how much they agree with each of 2 statements ("BLANK update messages are annoying." "BLANK update messages are confusing." and their answer to "Approximately how long after you see a $B L A N K$ update message do you wait to apply the update?" for the software packages asked about on the survey. 


\begin{tabular}{rcc}
\hline Software & Annoying & Confusion \\
\hline Windows & 0.40 & 0.31 \\
Apple PC & 0.38 & 0.26 \\
iPhone & 0.35 & 0.23 \\
Android & 0.22 & 0.24 \\
Firefox & 0.09 & 0.06 \\
Chrome & 0.07 & 0.11 \\
Explorer & 0.16 & 0.03 \\
Safari & 0.08 & 0.12 \\
MS Office & 0.07 & 0.07 \\
Acrobat & -0.02 & -0.01 \\
iTunes & 0.44 & 0.25 \\
WMP & -0.04 & -0.02 \\
QuickTime & -0.10 & 0.07 \\
VLC & -0.36 & -0.39 \\
Norton & 0.15 & 0.06 \\
MacAfee & 0.19 & 0.60 \\
Malwarebytes & 0.36 & 0.33
\end{tabular}

Table 8: Pearson's $\mathrm{r}$ value between responses to "Approximately how long after you see a $B L A N K$ update message do you wait to apply the update?" and each of 2 ratings provided by respondents providing how much they agree with the statement " $B L A N K$ update messages are..." Softwares with sample size $<15$ respondents (i.e., Linux, OpenOffice, and Libre Office) were omitted due to low sample number. Video Games are also omitted from correlation analysis since those questions do not ask about a specific software.

As can be seen, Microsoft Windows and Apple Laptop/Desktops both show similar correlations between how quickly the respondent says they usually apply the update after seeing the message and how annoying and confusing they rate the messages from those systems. Other software categories, such as media and security also contain some programs that have notable correlations with annoyance and confusion, but not all programs in those categories correlate.

Interestingly, the pattern does not hold at all for other categories, including those critical to security, such as the browser. When examining the responses more closely, it was apparent that for most software, most participants reported never updating (see Table 7). Thus, there was no correlation to be found for those softwares.

More replication will be required to confirm these patterns in the general population, but there are explanations that could account for this phenomenon. Operating systems are unlike other software applications in that they are closer to the hardware and deal with the computer in a less compartmentalized fashion. Thus, the OS is seen as more important to users due solely to the fact that they use them more often than other programs and that most users do not install operating systems on their machine, since they commonly come pre-installed. This relationship, not shared by other types of software, may make users more aware of the importance of updating regularly. Also, many users are aware of the 
need for protection against malware, so it also makes sense that update delays are lower for some of those softwares as well. Beyond this, it's possible that update mechanism and software policy (e.g., requiring users to apply updates, allowing users to turn updates on/off) are partly responsible for some of the update delay distributions.

According to both the C-HIP and ARI models, differences in perception based on type of software could alter how the update messages must be designed to effectively convince users. In the C-HIP model, differences in perception reflect a difference in attitudes and beliefs (as they relate to the Attitudes/Belief stage) for different software. Thus, in order to align with different attitudes/beliefs, messages may have to be designed differently. Using the ARI model, differences in perception could alter how rational and emotional appeals affect the person when they are received. Thus, altered appeals may be needed for different software.

The connection between annoyance, confusion and update speed follows with a finding from a recent study that demonstrated a link between text complexity of messages and the attention given to the messages [14]. It then makes sense that many of the respondents in our sample who were annoyed and especially confused by messages would be less likely to apply them. As [14] showed, in some cases, they were not giving the messages enough attention.

\subsection{Prevalence of Annoyance and Confusion}

One strong trend in the responses is the tendency for participants to be annoyed by update messages. Respondents also indicated that they have commonly been confused by update messages.

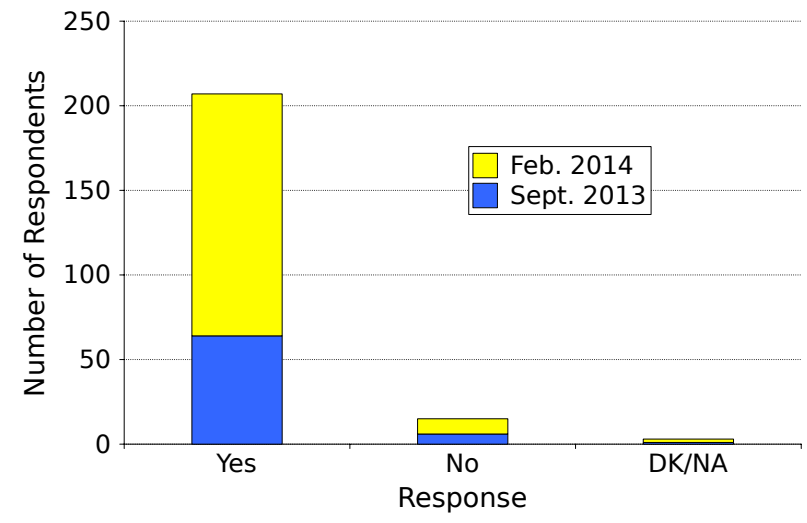

Figure 3: Responses to "Have you ever been annoyed by an update message?" Note: DK = Don't Know, NA = No Answer. 
Figure 3 summarizes the results for the question, "Have you ever been annoyed by an update message?" from both phases. About $92 \%$ of all respondents from the first phase of our study report having been annoyed by an update message. $93 \%$ of respondents from the second phase answer the question "Yes." This reflects a general negative sentiment in sampled users' minds regarding update messages.

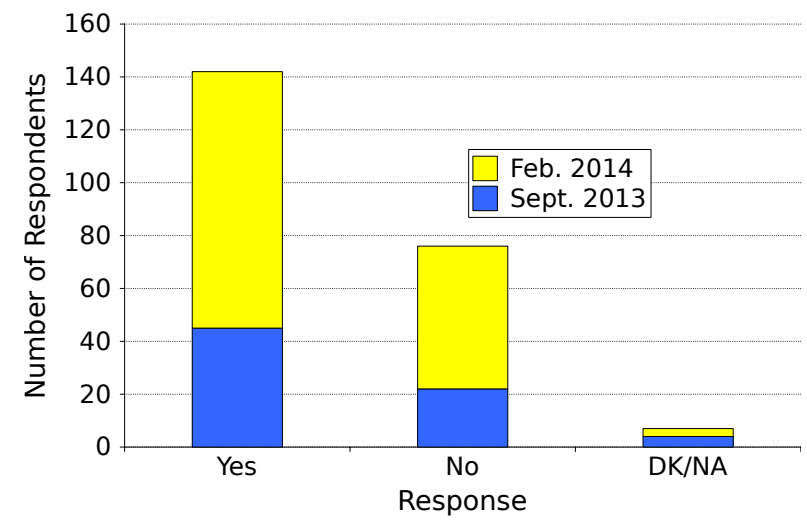

Figure 4: Responses to "Have you ever been confused by an update message?" Note: DK = Don't Know, NA = No Answer.

Figure 4 shows the results for the question about confusion. Over $63 \%$ of survey respondents in Sept. of 2013 report having been confused by an update message in the past. The same percentage of participants from the second round report having been confused.

Though respondents report common experiences of annoyance and confusion, there is significant variance in how annoying and confusing participants rated messages when asked to consider messages only from specific software packages as shown in Table 9. When aggregating the responses for each software package, the average annoyance rating is approximately 3.3 and the average confusion rating is 2.6 on the range of 1 to 7 .

Interestingly, some software packages' messages are rated as significantly more annoying than average. iTunes, for example, receives an average annoyance rating of 4.3. Windows tops that with an average annoyance rating of 4.5. Norton software and Apple Laptop/Desktop OS both also score high in annoyance with averages of 4.0 for each.

Participants report Microsoft Windows' update messages to be more confusing with an average of 3.1. Norton software garners an above average confusion rating at 3.3, the highest confusion average of softwares tested and considered. Of course not all software is rated more annoying/confusing than average. Chrome updates have a 2.4 average for annoyance, the lowest in the sample. 


\begin{tabular}{rcc}
\hline Software & Avg "annoying" & Avg "confusing" \\
\hline Windows & 4.5 & 3.1 \\
Apple PC & 4.0 & 2.8 \\
iPhone & 3.6 & 2.5 \\
Android & 3.3 & 2.6 \\
Firefox & 3.4 & 2.5 \\
Chrome & 2.4 & 2.0 \\
Internet Explorer & 3.5 & 3.1 \\
Safari & 2.5 & 2.3 \\
MS Office & 3.1 & 2.6 \\
Acrobat & 3.7 & 2.5 \\
iTunes & 4.3 & 2.8 \\
WMP & 2.9 & 2.6 \\
QuickTime & 3.5 & 2.6 \\
VLC & 2.6 & 1.9 \\
Norton & 4.0 & 3.3 \\
MacAfee & 3.2 & 2.3 \\
Malwarebytes & 2.7 & 2.0
\end{tabular}

Table 9: Average rating (from 1 to 7 ) of how much participants agree with the statement "BLANK update messages are..." with $B L A N K$ being replaced by each software and "..." being replaced by "annoying" or "confusing." Softwares with sample size $<15$ respondents (i.e., Linux, OpenOffice, and Libre Office) were omitted due to low sample number. Video Games are also omitted from this analysis since those questions do not ask about a specific software.

Malwarebytes has an average confusion of 2.0, representing the lowest average confusion score of considered software.

Other work has found similar occurrences of annoyance and confusion with updates [30,29]. In one study, the authors identified that participants commonly did not understand what was being changed in their computer based on the messages, indicating confusion with the messages, which, as seen above, our sample also reported. The other report showed that participants in their study had trouble understanding why updates were important and that lead them to not apply them [29]. In some case, participants in that study showed large amounts of frustration when they did not understand an update's importance. This frustration could be the annoyance with updates that our participants are reporting.

Having been annoyed in the past will make a user less likely to adhere in the future, as predicted by both behavioral models. Making update messages less invasive while still being noticeable and effective is key to increasing acceptance rates of software updates. Like annoyance, confusion not only hurts the message being misunderstood, but also future update messages due to the influence of past experiences, as explained in the C-HIP model. It is important for users to understand the messages they read so that they can make informed decisions. If users are confused, they are more likely to reject the recommended updates. 
According to the ARI model, annoyances are undesirable as annoyance can become a negative emotional argument against applying updates. For example, if an update annoys a user, they may turn off the notification mechanism in the software. In the context of the C-HIP model, annoyance would affect the Attitudes-Beliefs stage in the cognition and information processing in a negative way and may lead towards non-compliance. Similarly, confusion in the ARI model hurts the rational argument, and maps to the comprehension stage in the C-HIP model. According to both models, confusion is likely to lead towards non-compliance.

\subsection{Negative Feelings Towards Software Companies}

Overall, respondents from both phases are quite cynical when it comes to why they thought companies released software updates. Figure 5 shows that, in the first round, $63 \%$ of participants answer "To stay competitive against other software choices." The frequency of this response rises to $80 \%$ for respondents to the second round survey. Only 3 of 71 surveyed users chose the response "They care about users." in the first round and 7 or 152 in the second round.

Part of emotional persuasion, according to the ARI model, is a general positive emotional impression of the source (e.g., software company). If users feel that the company releases software updates because they care about them, users are more likely to apply the update. This impression of caring does not have to be applied as literally as convincing users that they are in the CEO's direct consideration, but rather, feelings that the company cares can be increased with a generally better public image. If people like a company, they will be more likely to adhere to warnings from them because they will have more trust in the company.

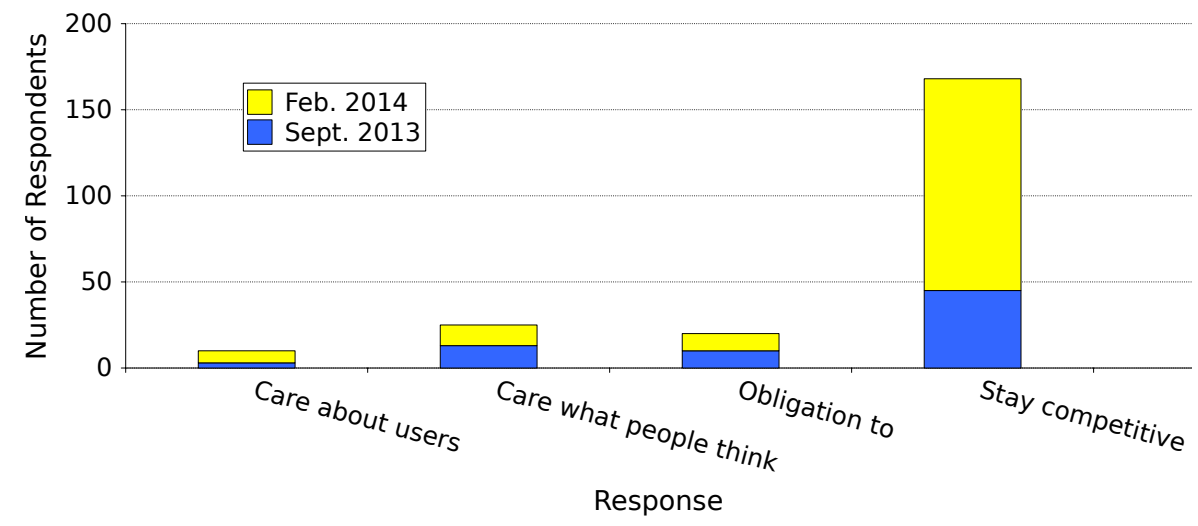

Figure 5: Responses for "What do you think is the PRIMARY motivation for software companies to release software updates?" 


\begin{tabular}{rcc}
\hline Software & Number of Respondents & Average Opinion Rating \\
\hline Microsoft Windows & 114 & 5.7 \\
Apple Desktop/Laptops & 57 & 5.9 \\
Linux & 11 & 5.9 \\
iPhone & 80 & 6.0 \\
Android & 33 & 5.8 \\
Firefox & 53 & 5.2 \\
Chrome & 119 & 6.1 \\
IE & 30 & 4.1 \\
Safari & 50 & 4.9 \\
MS Office & 135 & 5.9 \\
Open Office & 9 & 4.8 \\
Adobe Acrobat & 61 & 5.1 \\
Libre Office & 5 & 4.0 \\
iTunes & 107 & 5.2 \\
WMP & 51 & 4.7 \\
QuickTime & 25 & 4.2 \\
VLC & 35 & 5.5 \\
Skype & 90 & 5.2 \\
Norton & 31 & 5.1 \\
McAfee & 33 & 5.0 \\
Malwarebytes & 16 & 5.8
\end{tabular}

Table 10: Average opinion ratings (from 1 to 7 ) for all software packages asked about in the extended survey.

Despite this cynicism, most software packages are rated favorably in the extended study data. Participants were asked to rate their overall opinion of each software they commonly used on a scale of 1 to 7 with 1 being very negative and 7 being very positive. All software packages have an average rating greater than 4.5 except Internet Explorer, Libre Office, and QuickTime. Only 3 other programs are rated, on average, under 5. They were Safari, OpenOffice, and Windows Media Player. In all, we asked about 21 possible software packages. The total number of respondents and average opinion ratings for all software are shown in Table 10.

The general positive views of the software we asked about can be explained by the fact that we asked participants to tell us about software they commonly use. It should come as no surprise that the software people commonly use is rated highly by them. If they thought little of the software, they probably would not use it and therefore would not complete one of our surveys. That said, positive views of software are good for update compliance since it creates a positive opinion of the software and update in the mind of users. Thus, it is important to update compliance that software companies maintain the positive opinions of their users.

The positive view of commonly used software is an interesting finding when 
put in the context of [29] which showed that participants were more likely to update more commonly used software. The ARI model states that if someone has a positive association with the source of a message, they are more included to heed that messages. The results of our study and prior work could showing this effect at work. It's possible that [29]'s participants are more likely to update software they commonly use because they like those software more, as was the case with our participants.

\subsection{Image Based Survey Results}

Participants in the second round of our study were asked to evaluate real world update messages. They were shown 14 images in series and asked to rate how important, annoying, confusing, and noticeable they thought each message was. They were also invited to express what they liked and didn't like about each message in a comment format. Our key findings are below.

First, in our data, we found that noticeability and importance are correlated. This indicates that when users rated something as important, they also rated it as being noticeable. In all responses, Pearson's $r$ value for the responses to the questions "How important is the message?" and "How noticeable is the message?" is 0.42 .

Please note that noticeability of a message has been shown to be very important to the ability of that message to transmit information to a reader according to both the ARI and C-HIP models. Our findings indicate that it is also tied into the perceived importance of a message. This relationship can work in either direction. A more noticeable message may look more important and a message that is more important may be more easily noticed by the user. Hence, designers must focus on creating noticeable messages, which may in turn help influence users see a message as more important.

The study conducted for [8] showed a similar connection between importance and noticeability. Specifically, they found that some participants had cared about Android permissions in the past, but lost concern over time due to seeing permission too often [8]. As suggested by the correlation between noticeability and importance in our data, this also suggests that how important a messages is to someone affects how noticeable it is to them.

Second, similar to the important/noticeable relationship, there is also a correlation between participants' ratings of how confusing a message is and how annoying it is. The $\mathrm{r}$ value for responses to the questions "How annoying is the message?" and "How confusing is the message?" is 0.50. Like before, this relationship can work both ways. A confusing message could be frustrating, and thus annoying, and an annoying message may be hard to focus on and understand, thus making it confusing. Being annoyed or confused by a message are both undesirable responses in the context of both the ARI and C-HIP models.

With these facts, we devised a simple ranking metric to help determine how the images were perceived overall, relative to each other. To do this, we normalized the average response for each question, from each image (we divided each average response by 7 ). Since importance/noticeability were correlated 


\begin{tabular}{cccccr}
\hline Image \# & Important & Annoying & Confusing & Noticeable & Ranking \\
\hline 04 & 3.6 & 4.5 & 4.2 & 4.1 & -0.15 \\
06 & 4.3 & 4.4 & 4.0 & 4.5 & 0.06 \\
14 & 3.0 & 3.8 & 2.2 & 3.4 & 0.06 \\
10 & 3.9 & 4.2 & 4.1 & 4.9 & 0.07 \\
13 & 3.5 & 4.0 & 3.6 & 4.7 & 0.07 \\
07 & 3.6 & 4.3 & 3.0 & 4.8 & 0.13 \\
01 & 3.7 & 3.5 & 3.4 & 4.6 & 0.18 \\
08 & 4.6 & 3.9 & 3.5 & 4.8 & 0.28 \\
03 & 3.6 & 3.8 & 2.5 & 4.9 & 0.29 \\
09 & 3.6 & 3.4 & 2.3 & 4.8 & 0.33 \\
12 & 3.8 & 3.3 & 2.3 & 5.0 & 0.39 \\
02 & 5.4 & 4.3 & 3.1 & 6.0 & 0.51 \\
05 & 5.9 & 4.1 & 3.6 & 6.3 & 0.58 \\
11 & 4.8 & 2.8 & 2.2 & 4.8 & 0.61 \\
Average & $\mathbf{4 . 1}$ & $\mathbf{3 . 9}$ & $\mathbf{3 . 1}$ & $\mathbf{4 . 8}$ & $\mathbf{0 . 2 4}$
\end{tabular}

Table 11: Overall image response statistics, ordered by ranking to highlight range. Please see the Appendix for the list of images.

and annoyance/confusion were correlated, we considered these two groupings by subtracting the sum of the normalized annoyance and confusion values from the sum of the importance and noticeability values. For example, the average response rates can be seen in Table 11. For Image (2), the ranking was calculated by computing $((5.4 / 7)+(4.3 / 7))-((3.1 / 7)+(6.0 / 7))=0.51$. These rankings help identify the following findings.

\subsubsection{The "Best" and the "Worst"}

As per our ranking scheme described above, image (11) is the most highly ranked image with a ranking metric of 0.61 compared to an average ranking metric of 0.24. This image can be seen in Figure 6. This image has high ratings of importance and noticeability coupled with low ratings for annoyance and confusion. According to both the C-HIP and ARI models, this is a very desirable set of traits. A noticeable message can sometimes be annoying, but this image seems to balance these two traits for our participants. A low confusion rating and a high importance ranting means users will be both more likely to understand the message's content and to understand the need to apply the message promptly, since it is perceived as important.

On the other end of the spectrum is image (4), which had the lowest ranking at -0.15 . The image can be seen in Figure 7. Opposite image (11), image (4) has low noticeability and importance ratings as well as high confusion and annoyance ratings.

Looking at image (11) (Figure 6) and image (4) (Figure 7), some possible clues into message design become apparent. Image (11) is from iOS, which is the operating system for Apple's iPhone. This message has an average annoyance and confusion rating of 2.9 and 2.3 respectively, whereas the aggregate average 


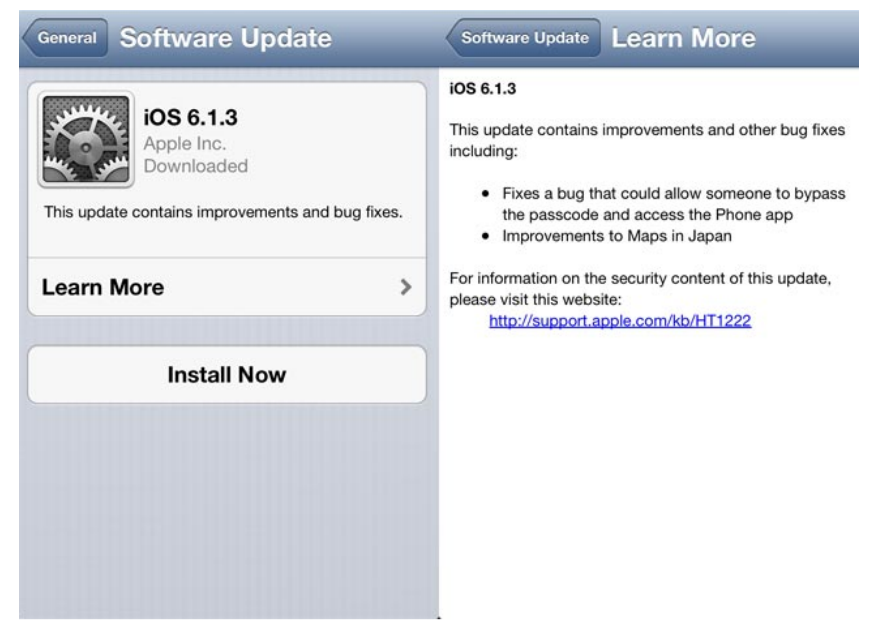

Figure 6: Image (11) from Table 7 shown to participants during the image based survey.

annoyance rating for all images is 3.9 and the aggregate average for confusion is 3.2. The color scheme of this message is muted, information is clear and easy to follow, and users are given a clear place to proceed to install the updates. All of these features, according to the C-HIP and ARI models, can influence people to see the message as less annoying and make the message easier to understand, thus reducing confusion as well.

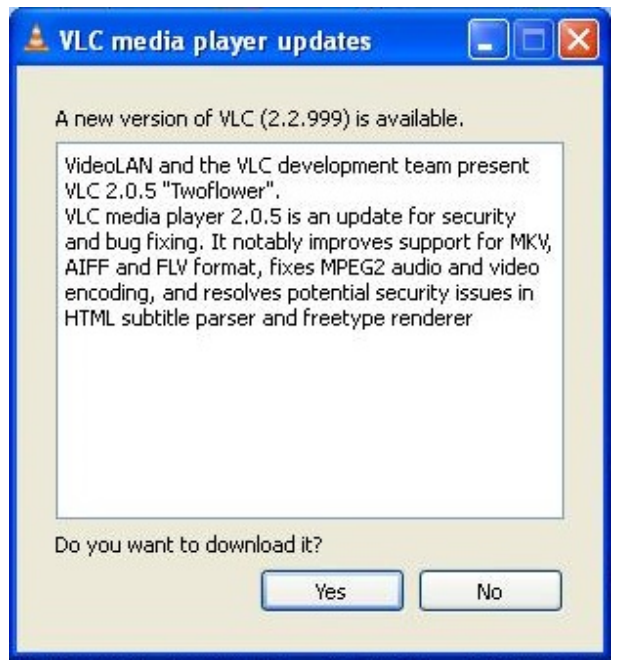

Figure 7: Image (4) from Table 7 shown to participants during the image based survey.

In contrast, image (4) (Figure 7) shows the image with the highest aggregate averages for annoyance and confusion which are 4.5 and 4.2 respectively. This 
message is from VLC running on a Windows XP computer. Unlike the image in Figure 6, the information in this message is formatted poorly, hard to read, and technical. These factors can increase the level of confusion caused by the message, as predicted in the affective-cognitive models. They may also increase the level of annoyance due to frustration.

\subsubsection{Importance and Noticeability}

We draw attention to two specific images. First is a fake update message for the Firefox browser. It is important to note that this message is not real, but was designed to look real. The second image is a virus notification for an Apple PC anti-virus software. These images ranked highest in both noticeability and importance as seen in Figures 8 and 9 .

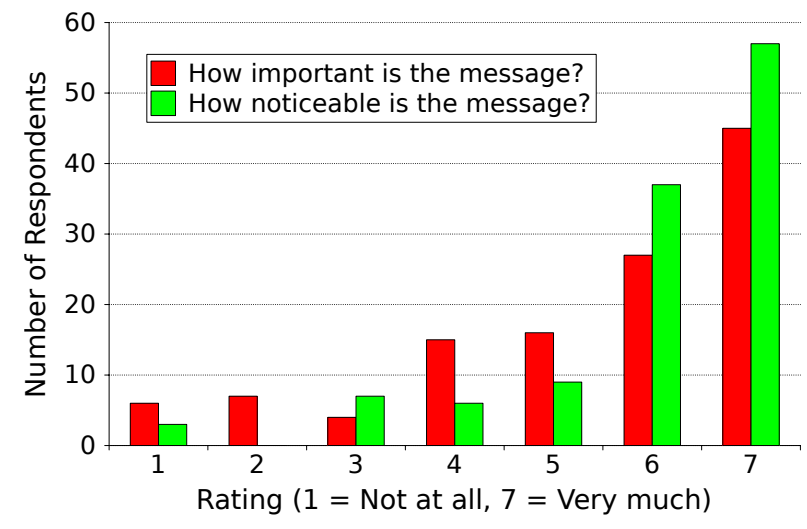

Figure 8: Response frequencies for image(2) in Table 7

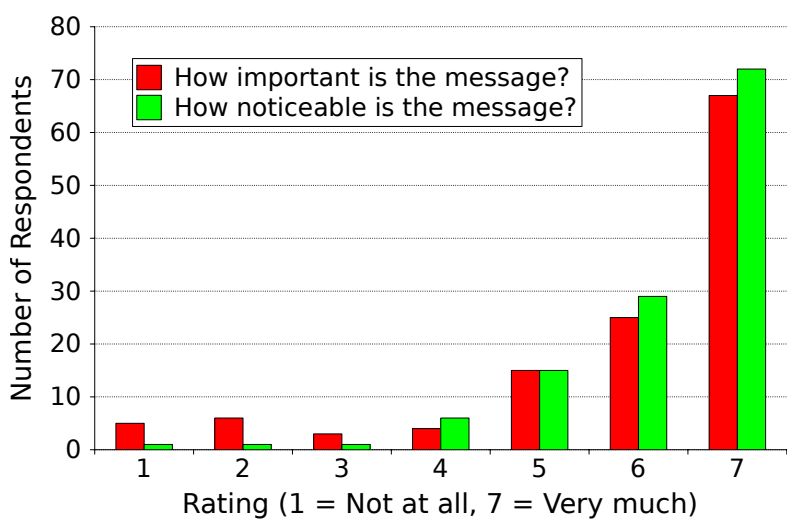

Figure 9: Response frequences for image(5) in Table 7

Table 11 summarizes the responses for the update messages discussed. These messages can be used as models to help increase noticeability and perceived 
importance of other update messages. In both cases, the images are colorful, using either bold reds or contrasting blacks and yellows. The fact that one is from an anti-virus program and the other from malware shows that these concepts have been applied for other goals and can be employed in update message design.

Both the C-HIP and ARI models agree that a message being noticeable and registering as important to a person is important to the person adhering to the warning. Thus, it is desirable to design update/waring messages that are noticeable to users and that they will think are important.

\section{Discussion}

While the number of participants in our study is not large, and one round of the survey was uncompensated while the other was compensated, we would like to point out that the responses for common questions between both survey rounds (i.e., the short survey questions) from both phases showed similar distributions for each question. Figure 10 shows the normalized frequencies from both phases for three of the questions from the short survey.

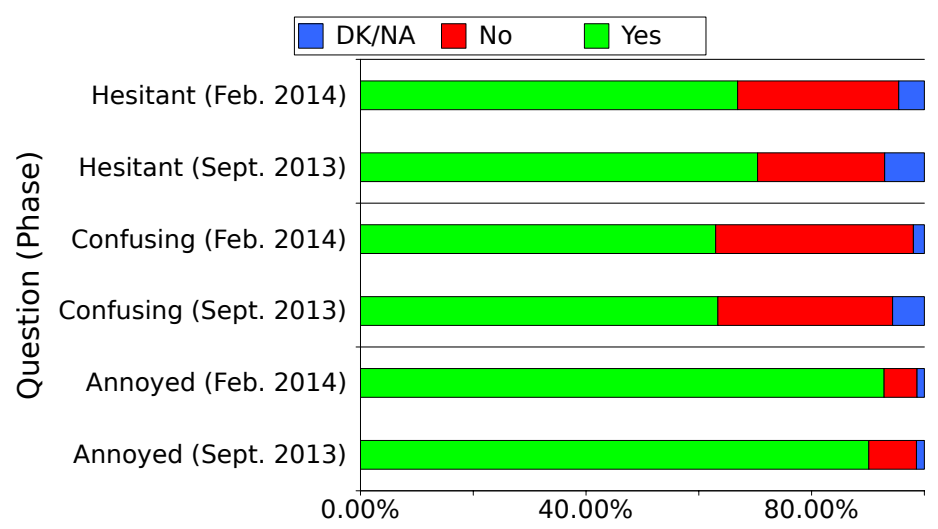

Figure 10: Normalized response frequencies for three Yes/No questions from the short survey.

The three questions shown are "Have you ever been hesitant to apply an update?", "Have you ever been annoyed by an update message?", and "Have you ever been confused by an update message?" . Though the nominal number of "yes" and "no" responses was different between the two samples due to the different sample sizes, when the response frequencies are normalized to $100 \%$, it is clear that the response rates for each question are very similar between samples. This followed for all of the questions from the short survey, as can be seen in evidence from the short survey presented in the Evaluation section. 
While we are unable to extrapolate our findings to the population in general due to relatively small sample size, the striking similarities in response patterns between the two samples despite the differences in sample size, sample demographics, age distribution, and compensation scheme indicates that the study findings is likely to hold for a larger population as well.

Overall, our study successfully identified the prevalence of negative impressions commonly associated with software update messages, which leads towards non-compliance. We strongly believe that this work is a starting point for a larger overall investigation into the problem of update message design, and will provide interesting leads for investigators to explore and design better and effective software warning/update messages in future. One route of future work includes more extensive, deeper, numerical investigations into what aspects of update messages are more important than others and how can messages be designed harnessing these components to better persuade users.

\section{Conclusion}

In this paper, we perform a quantitative study of existing software update messages to identify some users' perceptions, experiences, and beliefs regarding software update mechanisms. Our results show that users are commonly annoyed and sometimes confused by update messages. More importantly, our studies show a general negative attitude associated with software warning and up-

date messages. We also identify that users' opinions regarding the software and the vendors often affect their decision regarding applying an update message. Finally, we relate all these findings to people's decision making processes when considering update messages leveraging two alternative models. We strongly believe that our results will foster future research in this critically important direction and will lead towards the design of better, more effective software update/warning messages and delivery mechanisms. 


\section{Appendix}

Images used in image based survey

\section{Image 1}

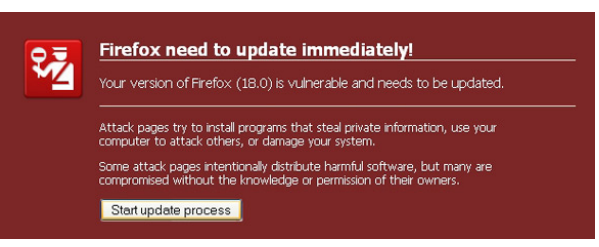

Image 2

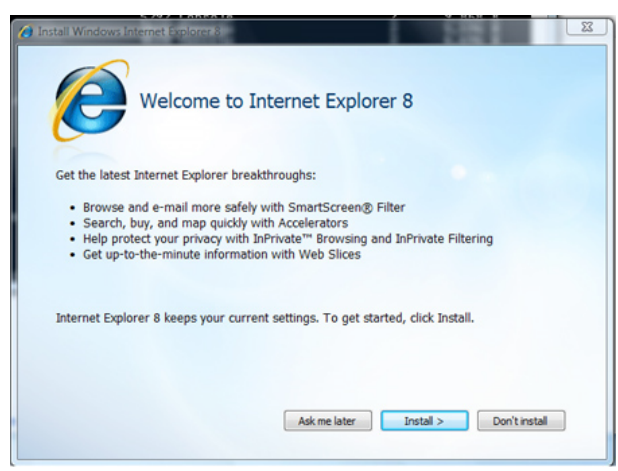

Image 3

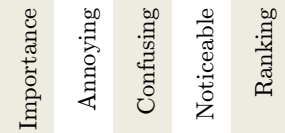

3.7

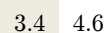

0.18

5.4

$\begin{array}{llll}4.3 & 3.1 & 6.0 & 0.51\end{array}$

$3.6 \quad 3.8$

$2.5 \quad 4.9$

0.29 
Images used in image based survey (continued)

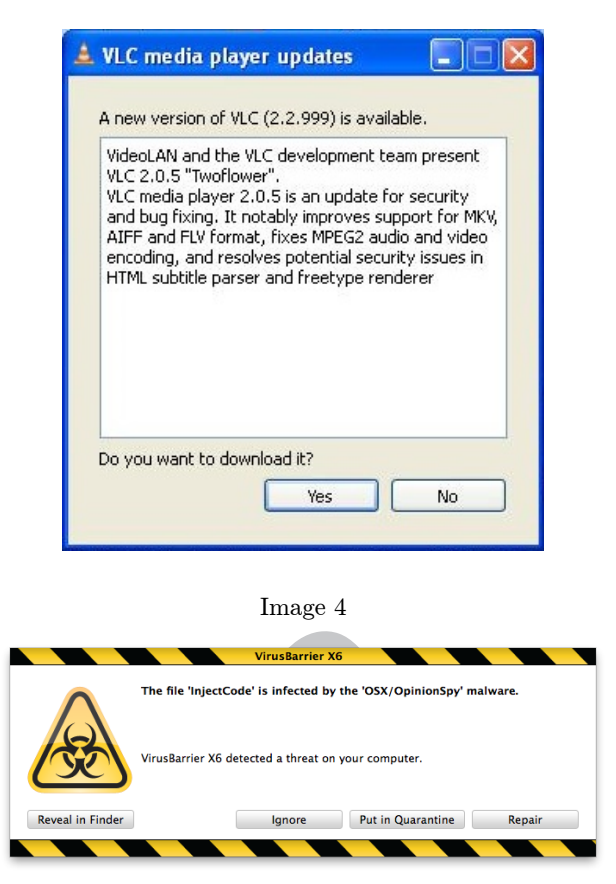

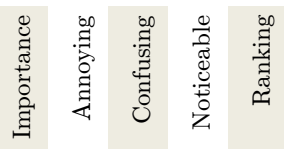

Image 5

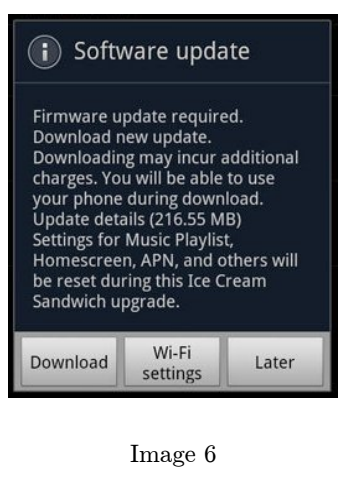

$\begin{array}{lllll}3.6 & 4.5 & 4.2 & 4.1 & -0.15\end{array}$

$\begin{array}{lllll}5.9 & 4.1 & 3.6 & 6.3 & 0.58\end{array}$

$\begin{array}{lllll}4.3 & 4.4 & 4.0 & 4.5 & 0.06\end{array}$ 
Images used in image based survey (continued)

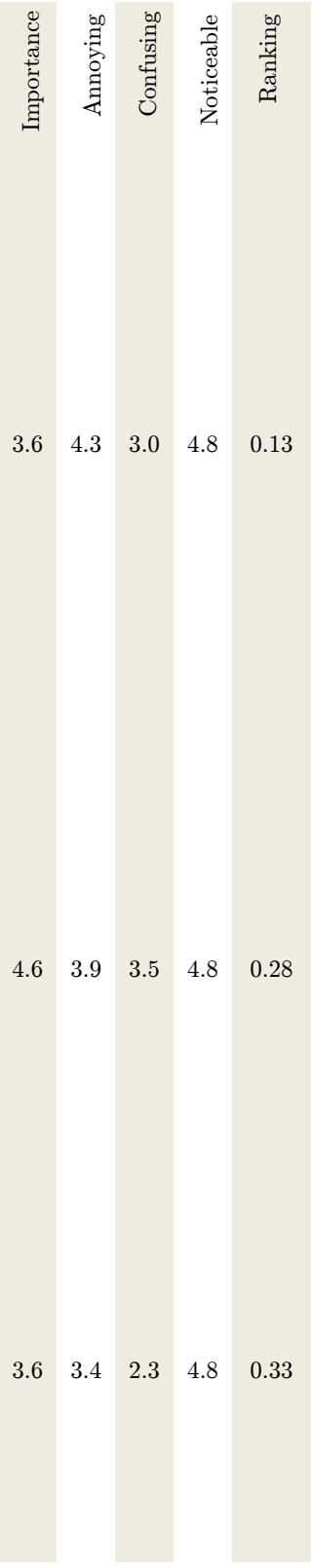


Images used in image based survey (continued)
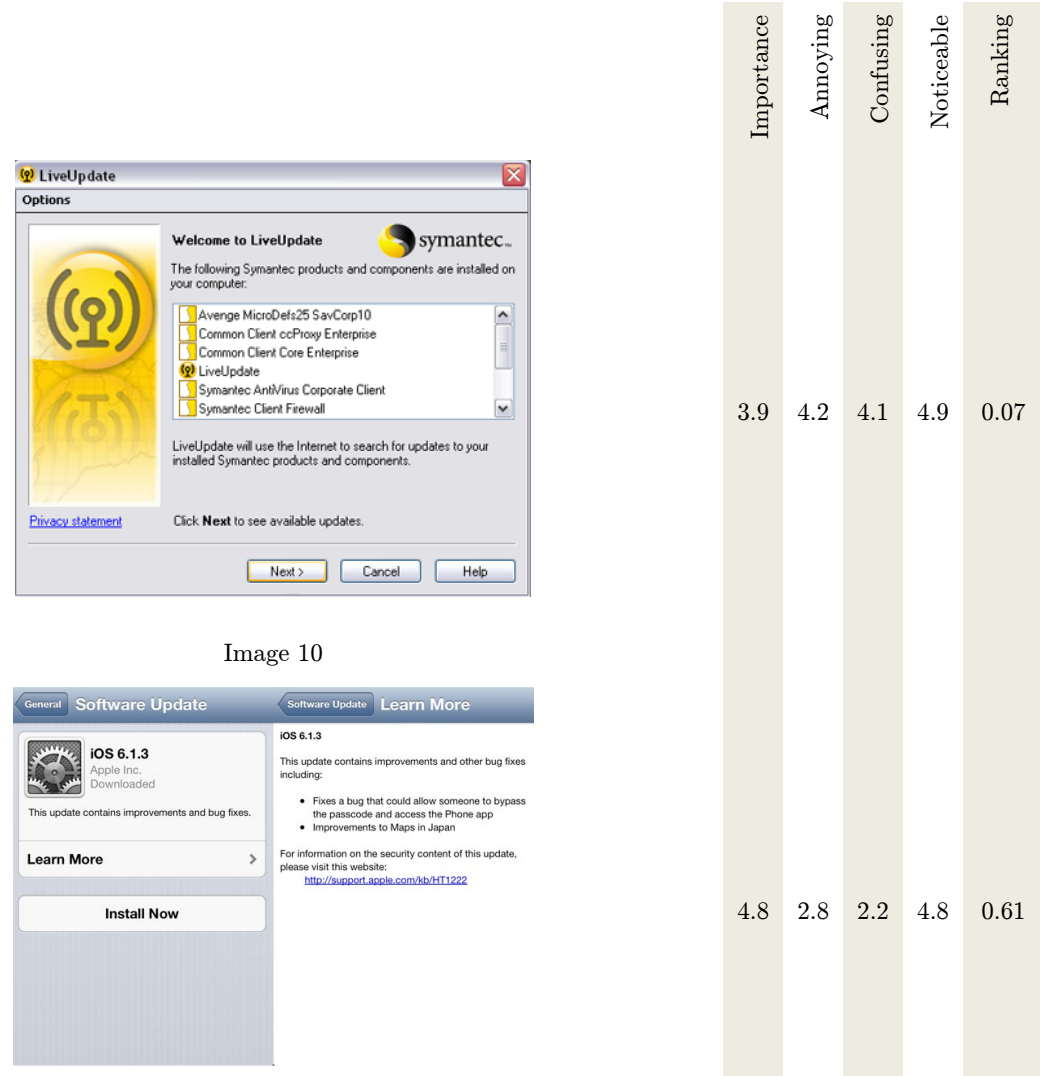

Image 11

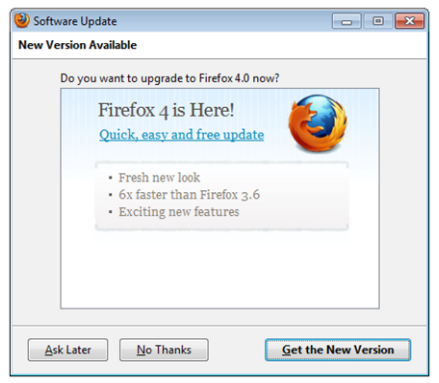

Image 12

3.9 $\begin{array}{llll}4.2 & 4.1 & 4.9 & 0.07\end{array}$

4.8

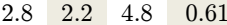

3.8 $\begin{array}{llll}3.3 & 2.3 & 5.0 & 0.39\end{array}$ 
Images used in image based survey (continued)
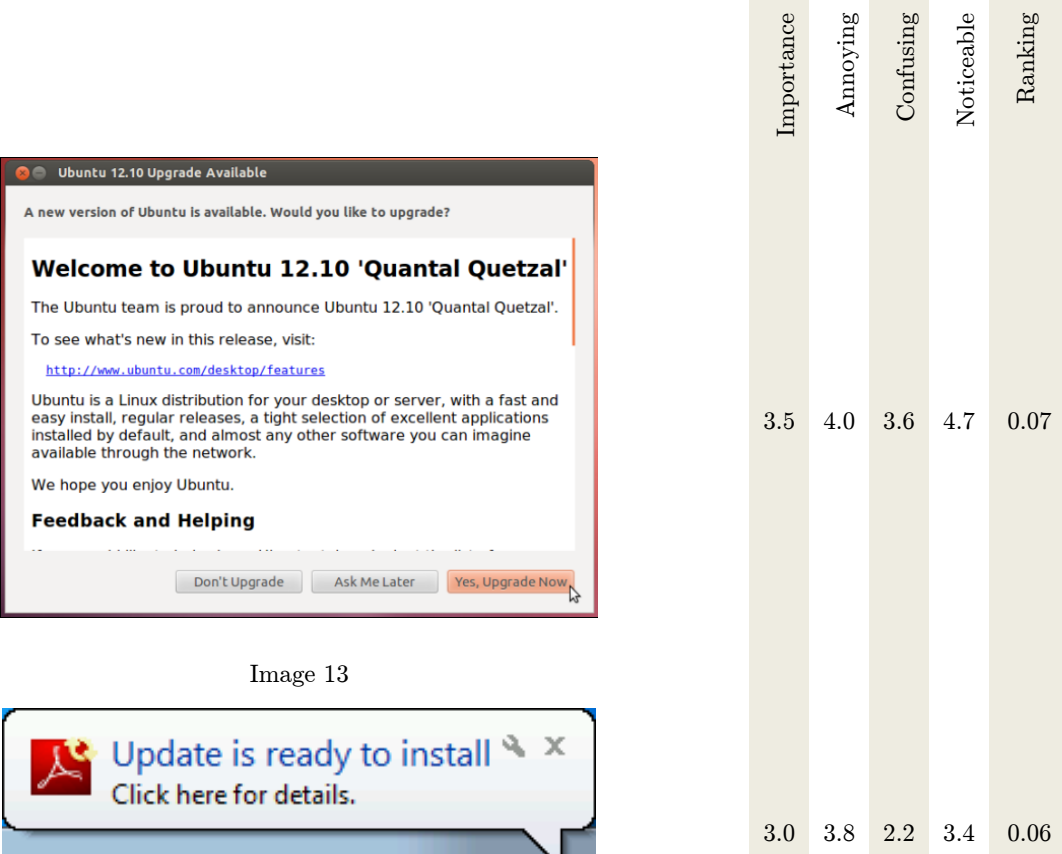

Image 14 
[1] Internet social networking risks. http://www.fbi. gov/about-us/investigate/counterintelligence/ internet-social-networking-risks. Accessed: 2013-09-18.

[2] Curt C Braun, Stephanie A Glusker, Ronda S Holt, and N Clayton Silver. Adding consequence information to product instructions: changes in hazard perceptions. In Proceedings of the Human Factors and Ergonomics Society Annual Meeting, volume 39, pages 346-349. SAGE Publications, 1995.

[3] Ross Buck, Erika Anderson, Arjun Chaudhuri, and Ipshita Ray. Emotion and reason in persuasion: Applying the ari model and the casc scale. Journal of Business Research, 57(6):647-656, 2004.

[4] Norton by Symantec. Why security updates are vital. http://us.norton . com/vital-security/article. Accessed: 2014-08-01.

[5] Vincent C Conzola and Katherine W Klein. Need for cognition: an individual differences approach to understanding warning effectiveness. In Proceedings of the Human Factors and Ergonomics Society Annual Meeting, volume 42, pages 811-815. SAGE Publications, 1998.

[6] Vincent C Conzola and Michael S Wogalter. A communication-human information processing (c-hip) approach to warning effectiveness in the workplace. Journal of Risk Research, 4(4):309-322, 2001.

[7] Judy Edworthy and Austin S Adams. Warning design: A research prospective. CRC Press, 1996.

[8] Adrienne Porter Felt, Elizabeth Ha, Serge Egelman, Ariel Haney, Erika Chin, and David Wagner. Android permissions: User attention, comprehension, and behavior. In Proceedings of the Eighth Symposium on Usable Privacy and Security, page 3. ACM, 2012.

[9] J Paul Frantz. Effect of location and presentation format on attention to and compliance with product warnings and instructions. Journal of safety research, 24(3):131-154, 1993.

[10] Daryle Jean Gardner-Bonneau, Fawzi Kabbara, Minjohn Hwang, Hans Bean, Marilyn Gantt, Kevin Hartshorn, Jennifer Howell, and Rahim Spence. Cigarette warnings: Recall of content as a function of gender, message context, smoking habits and time. In Proceedings of the Human Factors and Ergonomics Society Annual Meeting, volume 33, pages 928930. SAGE Publications, 1989.

[11] Leo A Goodman. Snowball sampling. The annals of mathematical statistics, pages $148-170,1961$.

[12] Chris Grier, Lucas Ballard, Juan Caballero, Neha Chachra, Christian J Dietrich, Kirill Levchenko, Panayiotis Mavrommatis, Damon McCoy, Antonio 
Nappa, Andreas Pitsillidis, et al. Manufacturing compromise: the emergence of exploit-as-a-service. In Proceedings of the 2012 ACM conference on Computer and communications security, pages 821-832. ACM, 2012.

[13] Marian Harbach, Sascha Fahl, Thomas Muders, and Matthew Smith. Towards measuring warning readability. In Proceedings of the 2012 ACM conference on Computer and communications security, pages 989-991. ACM, 2012.

[14] Marian Harbach, Sascha Fahl, Polina Yakovleva, and Matthew Smith. Sorry, i dont get it: An analysis of warning message texts. In Financial Cryptography and Data Security, pages 94-111. Springer, 2013.

[15] Marian Harbach, Markus Hettig, Susanne Weber, and Matthew Smith. Using personal examples to improve risk communication for security \& privacy decisions. In Proceedings of the 32nd annual ACM conference on Human factors in computing systems, pages 2647-2656. ACM, 2014.

[16] Marian Harbach, Markus Hettig, Susanne Weber, and Matthew Smith. Using personal examples to improve risk communication for security \&\#38; privacy decisions. In Proceedings of the 32Nd Annual ACM Conference on Human Factors in Computing Systems, CHI '14, pages 2647-2656, New York, NY, USA, 2014. ACM.

[17] Curtis P Haugtvedt and Richard E Petty. Personality and persuasion: Need for cognition moderates the persistence and resistance of attitude changes. Journal of Personality and Social Psychology, 63(2):308, 1992.

[18] Markus Jakobsson and Steven Myers. Phishing and countermeasures: understanding the increasing problem of electronic identity theft. Wiley.com, 2006.

[19] Paul B Kline, Curt C Braun, Nancy Peterson, and N Clayton Silver. The impact of color on warnings research. In Proceedings of the Human Factors and Ergonomics Society Annual Meeting, volume 37, pages 940-944. SAGE Publications, 1993.

[20] Katherine McCoy. Information and persuasion: Rivals or partners? Design Issues, 16(3):80-83, 2000.

[21] Donald A Norman and Andrew Ortony. Designers and users: Two perspectives on emotion and design. In Proc. of the Symposium on Foundations of Interaction Design at the Interaction Design Institute, Ivrea, Italy, 2003.

[22] Srivatsan Parthesarathy, Reiner Fink, Sean L Flynn, and Ray Sun. Software update notification, March 5 2002. US Patent 6,353,926.

[23] Isabel Ramos and Daniel M Berry. Is emotion relevant to requirements engineering? Requirements Engineering, 10(3):238-242, 2005. 
[24] Mark Keith Reha and Charles F Morris. Software update manager, August 28 2001. US Patent 6,282,709.

[25] Juergen Sauer and Andreas Sonderegger. The influence of prototype fidelity and aesthetics of design in usability tests: effects on user behaviour, subjective evaluation and emotion. Applied ergonomics, 40(4):670-677, 2009.

[26] Steve Sheng, Bryant Magnien, Ponnurangam Kumaraguru, Alessandro Acquisti, Lorrie Faith Cranor, Jason Hong, and Elizabeth Nunge. Antiphishing phil: the design and evaluation of a game that teaches people not to fall for phish. In Proceedings of the 3rd symposium on Usable privacy and security, pages 88-99. ACM, 2007.

[27] RJ Sojourner and MS Wogalter. The influence of pictorials on the comprehension and recall of pharmaceutical safety and warning information. International Journal of Cognitive Ergonomics, 2(1/2):93-106, 1998.

[28] MIT Information Systems and Technology. Software patches and os updates. https://ist.mit.edu/security/patches. Accessed: 2014-08-01.

[29] Kami E. Vaniea, Emilee Rader, and Rick Wash. Betrayed by updates: How negative experiences affect future security. In Proceedings of the 32Nd Annual ACM Conference on Human Factors in Computing Systems, CHI '14, pages 2671-2674, New York, NY, USA, 2014. ACM.

[30] Rick Wash, Emilee Rader, Kami Vaniea, and Michelle Rizor. Out of the loop: How automated software updates cause unintended security consequences. pages 89-104. USENIX Association, 9999.

[31] Michael S Wogalter and John W Brelsford. Incidental exposure to rotating warnings on alcoholic beverage labels. In Proceedings of the Human Factors and Ergonomics Society Annual Meeting, volume 38, pages 374-378. SAGE Publications, 1994.

[32] Michael S Wogalter, Dave DeJoy, and Kenneth R Laughery. Warnings and risk communication. CRC Press, 1999.

[33] Michael S Wogalter and David A Dietrich. Enhancing label readability for over-the-counter pharmaceuticals by elderly consumers. In Proceedings of the Human Factors and Ergonomics Society Annual Meeting, volume 39, pages 143-147. SAGE Publications, 1995.

[34] Michael S Wogalter, Michael J Kalsher, and Raheel Rashid. Effect of signal word and source attribution on judgments of warning credibility and compliance likelihood. International Journal of Industrial Ergonomics, 24(2):185-192, 1999.

[35] Michael S Wogalter, Bernadette M Racicot, Michael J Kalsher, and S Noel Simpson. Personalization of warning signs: the role of perceived relevance on behavioral compliance. International Journal of Industrial Ergonomics, 14(3):233-242, 1994. 
[36] Michael S Wogalter and Eric F Shaver. Evaluation of list vs. paragraph text format on search time for warning symptoms in a product manual. Advances in Occupational Ergonomics and Safety, 4:434-438, 2001.

[37] Weidong Xia and Gwanhoo Lee. The influence of persuasion, training and experience on user perceptions and acceptance of it innovation. In Proceedings of the twenty first international conference on Information systems, pages 371-384. Association for Information Systems, 2000.

[38] Stephen L Young and Michael S Wogalter. Relative importance of different verbal components in conveying hazard-level information in warnings. In Proceedings of the Human Factors and Ergonomics Society Annual Meeting, volume 42, pages 1063-1067. SAGE Publications, 1998.

[39] Gretchen P Zobel. Warning tone selection for a reverse parking aid system. In Proceedings of the Human Factors and Ergonomics Society Annual Meeting, volume 42, pages 1242-1246. SAGE Publications, 1998. 
- We distribute two surveys to gauge participants' attitudes and behaviors related to software updates and warnings.

- We use two human information processing models to design our study and analyze results.

- Participants report common annoyance, confusion, and hesitation with software update and warning messages.

- Some emotions (e.g., annoyance/confusion, noticeability/importance) were found to be correlated.

- We identify areas of improvement for communication of software warnings and updates. 